This is a pre-copyedited, author-produced PDF of an article accepted for publication in Feminism and Psychology following peer review. The definitive publisher authenticated version is forthcoming.

DOI: TBC

\title{
'Blindness to the obvious'?:
}

\section{Treatment experiences and feminist approaches to eating disorders}

\section{Su Holmes}

\section{Introduction}

Critical feminist work on eating disorders (EDs) has aimed to dismantle the idea that an 'absolute Truth of "anorexia" is possible', critiquing the notion that anorexia exists independently of the medical discourses through which it is constructed (Malson, 1998, p.189). In seeking to wrest the definition of anorexia - and EDs more widely - away from the province of medical discourse, feminist work has highlighted how EDs should be approached as an object of historical, discursive and intellectual struggle. But this struggle has historically taken place on an 'institutionally uneven' terrain (Gremillion, 2003, p. 27), especially with regard to treatment practices.

This is encapsulated in the opening to Helen Gremillion's book Feeding Anorexia: Gender and Power at a Treatment Center, when she relays an encounter with the head male psychiatrist at the impatient unit where she was undertaking her study. After explaining the influence of 'feminist therapies and theories' on her project, Gremillion describes how the psychiatrist smilingly dismissed her expertise with the assertion: 'We'll show you what anorexia is really about [emphasis in original]' (2003, p. xvii). Compare the psychiatrist's blithe dismissal with the words of a 19 year old woman as she articulated her experience of 
encountering feminist work on EDs for the first time. Speaking specifically of Susan Bordo's Unbearable Weight (1993) which she came across after the acute phase of her treatment for anorexia had passed, she recalls how:

[I felt like] this changes everything. It was like a real lightbulb moment... If anyone asked me 'what was the moment that changed your life?' it was sitting there reading this book. First I was shocked, then I was in tears, then I re-read it and it felt like someone had finally spoken from my side [original emphasis] (P12).

Of all the 'mental disorders' categorised in the Diagnostic and Statistical Manual of Mental Disorders, anorexia and bulimia are most explicitly described as being linked to 'culture' (2013, p. 342). But although EDs are now often approached as biopsychosocial problems, the social or cultural aspects of the equation are often marginalised in treatment - relegated to mere contributory or facilitating factors (Bordo, 1993; Burkitt, 2001; Warin, 2010). In contrast, the body of work which is variously called feminist, critical feminist, socio-cultural or culturalist is primarily concerned with the relationship between EDs and the social/ cultural construction of gender. With the first book in the British context often seen to be Susie Orbach's (1978) best-seller, Fat is a Feminist Issue, this work has something of a 35 year history. Many of the earliest feminist interventions were developed by women who were practising therapists or counsellors which, in addition to Orbach $(1978,1986)$ included Kim Chernin (1985), Marlene Boskind-White (1979), Marilyn Lawrence (1984) and Robyn Sesan (1994). As such, this work discussed feminist approaches in the context of practice, from the feminist therapist /client relationship to the possibility of feminist models for in-patient treatment. But if we fast forward to today, whilst feminist analyses of treatment practices 
continue to appear (Malson and Ryan, 2008; Boughtwood and Halse, 2010; Gremillion, 2003), feminist work often takes place at a considerable remove from treatment intervention.

Feminist research has offered crucial insights into how the subjectivities of those diagnosed with an ED are multiply articulated in relation to the western cultural discourses that constitute normative femininities (Malson, 2009, p. 137), including how such identities are constructed, produced and resisted within treatment settings. Yet the discourse analysis often undertaken by feminists (Bordo, 1993; McSween, 1995; Malson, 1998; Saukko, 2008) has largely been used to critique the biomedical model from a scholarly distance, in part reflecting how the medical frameworks have resisted perspectives which challenge their power.

\section{Research questions}

This article draws upon data from 15 semi-structured interviews with women who have experience of anorexia and/or bulimia in order to explore a series of interlocking themes concerning the relationship between gender identity and treatment. Whilst there has been little systemic analysis of the impact of feminist approaches in treatment, qualitative feminist research has demonstrated the centrality of gender to how girls and women talk about the aetiology and experience of an ED (MacSween, 1995; Malson, 1998, 2009; Burns, 2004; Saukko, 2008). In responding to the apparent disconnect between 'patient' and treatment here, this article thus seeks to explore three key questions. Firstly, what role did gender play in how the women understood the aetiology of their eating problems, and to what extent was this theme taken up by, or explored within, their treatment contexts? Second, when given a space to reflect on the feminist approaches to EDs, how did the participants evaluate such 
perspectives in relation to their own experiences of eating/ body distress, and their identities as women? Finally, to what extent did the participants perceive that the feminist perspectives should play a role in future ED treatment, and in what ways did they suggest that this might occur?

As this research was prompted by my own experience of encountering the feminist ED literature five years after I had recovered from anorexia, I also reflect on the interpersonal dynamics of the interview encounter - a topic that has been central to feminist qualitative research (Tang, 2002; Tracy, 2013). In particular, I consider how the entanglement of my identities - as woman, former 'anorexic', feminist and academic - were implicated within the research process, and the nature of the qualitative data which emerged.

\section{Literature review: Feminist approaches to eating disorders}

Anorexia and bulimia have historically been perceived as gendered problems, primarily affecting girls and young women in Western societies. Although the last fifteen years or so has seen a rise in the diagnosis of EDs in the male population (estimations of numbers vary here) (see Strother et al, 2012), gender is seen to remain the single biggest predictor of risk. In contrast to the biomedical emphasis on EDs as an individual pathology, feminist perspectives have sought to frame such problems within the social and cultural construction of gender and the expectations surrounding female bodies, appetite, sexuality and social roles.

The early feminist authors - writing just after Second Wave feminism and in Western contexts that were witnessing a considerable rise in EDs - linked such problems to the consequences of the Women's Movement and the resulting contradictions and pressures 
surrounding the female role (Chernin, 1985; Orbach, 1986). The significance of the media in propagating thinness as beauty was certainly here (Orbach, 1986; Wolf, 1991; Bordo, 1993), and a plethora of subsequent ED research has explored the degree to which media constructs are implicated within cultures of body/ eating distress (see summaries in Holstrom, 2004; and Stice et al, 2008). At the same time, the later feminist research has also been wary of overemphasising 'the inscriptive power of cultural images of thinness', or even the characterisation of EDs as 'body image' problems (Malson, 2009, p. 124), and situate anorexia and bulimia as 'graphic cultural statement $[\mathrm{s}]$ ' about 'the "conditions of being a woman" in contemporary western cultures... and .... as expressive of a diverse range of sometimes contradictory societal values' which work to map out the normative parameters of the feminine (Ibid, p. 137). ${ }^{1}$ Indeed, in resisting a pathologising division between those who have/ do not have EDs, feminist work has often sought to position eating/ body distress on a continuum with the discourses and practices of normative femininity (Bordo, 1993; Wolf, 1991; Malson, 1998; Saukko, 2008). For example, a good deal of feminist work on EDs has explored the cultural conflation between eating and sexual desire, situating eating/body distress in relation to historical discourses on 'appropriate' expressions of female sexuality (Lawrence, 1984, Orbach, 1986, Bordo, 1993, Malson, 1998, Burns 2004). That is not to suggest, however, that critical feminist perspectives have theorised anorexia in particular as simply a repressive form of body discipline. Much feminist research, particularly that influenced by post-structuralism and Foucauldian theory, has foregrounded the extent to which anorexic bodies and practices can be understood as 'multiply constituted' in relation to cultural prescriptions which work to construct western femininity (Malson, 2009, p. 136-7), playing out discourses of containment and discipline, as well as embodied agency and resistance. 
As this summary suggests, critical feminist work has historically privileged the study of anorexia over bulimia, despite the fact that bulimia is up to three times more common than anorexia in the UK (NHS Choices, 2015). As the bulimic body may be at a normal weight, it appears to be more 'inscrutable' as a visual text, lending itself less readily to critical approaches that position the body as visualising the symbolic struggles of femininity (Squire, 2003; Burns, 2009). The relative invisibility of bulimia within the feminist literature then contributes to a cultural and medical hierarchy in which anorexia is (further) 'sanctioned as the morally... and socially superior illness' (Squire, 2003: p. 20; Burns, 2004; Walsh and Malson, 2010). Although there have been attempts to think about anorexia and bulimia together as complex embodied, affective and ideological expressions of femininity (Malson and Burns, 2009), such a hierarchy remains worthy of note, and it has shaped the recruitment of my sample (below).

The feminist work on EDs has arguably had more visibility in scholarly rather than mainstream treatment contexts and - as with feminist epistemologies more widely - it has not measured its interventions in ways which align with more 'objectivist view[s] of science' (Skoger and Magnusson, 2015, p. 490). The books by feminist therapists are certainly replete with stories about how individual client narratives were productively elucidated (and assisted) by a range of feminist treatment models (Orbach, 1978; Chernin, 1985; Lawrence, 1984; Bloom et al, 1994), and there are of course feminist therapists still working in the field of ED treatment today. But it remains the case that there has been little systematic discussion about the success of such approaches, and certainly little published on other treatment possibilities, such as feminist discussion groups. Some of the early feminist literature referred to how the specialist ED clinics which emerged in the 1980s made attempts to address 'body image, women's issues and assertiveness training' (Sesan, 1994p. 253) as part of the multidimensional approach which moved beyond a singular focus on weight gain. In the UK 
context, there have also long since been examples such as the Women's Therapy Centre (WTC) - established in 1976 and co-founded by Susie Orbach - which seek to place and treat EDs within the context of women's cultural experience (see Bloom et al, 1994). ${ }^{2}$ But examples such as the WTC are the exception rather than the norm, and are unlikely to structure the treatment possibilities for many women diagnosed with an ED in the UK. Furthermore, the literature referencing in-patient programmes with a socio-cultural component was written in the 1980s (Levendusky and Dooley, 1985; Roth, 1986), and after this time, such discussions seem to disappear. Indeed, it is possible that the increasing turn toward more evidence-based approaches in ED treatment - with a clear example being the rise of Cognitive Behavioural Therapy (CBT) - that social/cultural concerns have been (further) marginalised.

In terms of the contemporary context, there has been an increasing practical and scholarly interest in the primary prevention of EDs, and such work has certainly invested in sociocultural understandings of body/ eating distress, particularly those relating to media influence (Piran, 2010; Levine and Maine, 2010). Although some scholars argue that the long-term impact of such interventions remains limited (Piran, 2010; Levine and Maine, 2010), others suggest considerably greater levels of success, providing impressive evidence from particular empirical examples (see Stice et al, 2009). Nevertheless, Niva Piran (2010) has argued that primary preventative programmes engage with socio-cultural understandings of eating disorders, especially the risk factor of gender, in limited ways. Rather than prioritising an approach which pivots on a critique of the slender media ideal as unrealistic and unhealthy (or which measures levels of perceptual 'distortion' in the subject), Piran argues that addressing gender as a risk factor in EDs involves exploring inequities of gender and their 'impact and expression' in all aspects of girls' social environments (2010, p. 185). This dovetails with the view expressed more widely in critical feminist approaches to EDs that 
whilst the internalisation of the slender ideal should certainly be seen as implicated within the development of body and eating distress (Bordo, 1993; Malson, 2009), anorexia and bulimia should not be reduced to problems of 'body image [emphasis in original]' (Malson, 2009, p. 136).

Finally, a related area of relevance - notwithstanding the critique above - is the qualitative research into the relationship between critical feminist identity and body image. A range of studies (see Rubin et al, 2004; Murnen and Smolak, 2009) have explored the extent to which critical feminist reflexivity may offer a form of protection from feelings of body dissatisfaction. Indeed, there is a considerable history of feminist perspectives being brought into dialogue with womens' experiences of embodiment, from the activism of the fat acceptance movement, to the explosion of discourses about 'body positivity' and 'body confidence' online (see Sastre, 2014). In terms of the academic research on feminism and body image, Murnen and Smolak (2009) suggest in their meta-analysis of the field that significant correlations have been found between a feminist identity and a lower drive for thinness. At the same time, the results of such studies are contradictory, in so far as although feminism is seen as offering the tools for criticizing cultural body ideals (and imagining new ones), it did not always 'neutralize the impact' of these discourses (Liimakka, 2013, p. 8). As Lisa Rubin et al suggest, participants in their study 'experienced a conflict between their feminist beliefs and their feelings... about their own appearance [original emphasis]' (2004, p. 27). Nevertheless, despite the assertion that such research has potentially important implications for both preventative work and treatment (Ibid; Murnen and Smolak, 2009), there is little evidence to suggest that these ideas have been evaluated in practice, or specifically in relation to EDs. Furthermore, despite the burgeoning nature of the relationship between popular feminism and experiences of body confidence/ distress online, the individualising thrust of biomedical approaches to EDs does little to encourage girls/women 
to understand their problem as part of wider ideologies relating to female appetite, body or desire.

\section{Methodology}

\section{Participants}

Ethical clearance for the project was granted by the University in April 2015, and participants were interviewed in Norwich (UK) in July/August the same year. Recruitment for the study was undertaken via the research page of Beat (the UK's national ED charity), and the subsequent circulation of the study via their social media. In addition to being female and 18 or over, the criteria for participating was that respondents had experienced treatment for anorexia and/or bulimia, and that they considered themselves to be recovered in this regard. Given that the feminist approaches explicitly critique mainstream treatments, it was felt unethical to recruit anyone who was currently in treatment, or who considered themselves to be struggling with their eating problem. A decision was also made not to limit the recruitment of participants to those with experience of anorexia in an effort to avoid wholly reinforcing the ways in which bulimia has often been marginalised within qualitative ED research.

The study recruited fifteen participants living in different regions of the UK: four with experience of bulimia, one with experience of bulimia and anorexia, one with experience of anorexia and binge eating disorder (BED), and nine with experience of anorexia. The ages of the participants ranged from 19-45, with the majority (11) bunching between the ages of 2434. Fifteen respondents was felt to be an appropriate number for the aims of the study given that I wanted to consider - in detail - their experiences of treatment, as well as the womens' evaluations of the feminist approaches, specifically in relation to their particular stories. In 
terms of ethnicity, eight respondents defined themselves as white British, two as white Scottish, one as white Northern Irish, one as white British Jewish, two as white American, and one as British Asian. The study thus clearly recruited a sample with a white bias, unfortunately reflecting and consolidating the prevalence of white voices in ED research, including those with a feminist inflection. A further bias was evident in terms of sexual orientation, with thirteen participants identifying as heterosexual, one as bisexual, and one as bi/pansexual. In addition, although respondents came from a range of socio-economic backgrounds, all had been to university, a factor that often emerged as relevant in their engagement with the feminist approaches we discussed.

\section{Procedure}

Participants took part in a one-to-one interview that lasted on average about one hour, and gave verbal consent for the interview to be recorded/ used for research purposes at the start. The interview schedule then covered key five areas including: 1) what forms of treatment participants had experienced and how they evaluated that treatment 2) whether they had a story/ stories about why they had developed an ED 3) the extent to which this story emerged from, or was addressed within, treatment 4) what they thought of some of the feminist approaches to understanding EDs 5) whether these would be productive or useful in treatment. Eliciting responses to themes 4-5 involved me sending the participants a summary sheet (comprised of two sides of written text) one week prior to the interview which outlined some of the ways in which critical feminist work had conceptualised EDs.

The sheet offered a brief outline of the ideas that have emerged from feminist postructuralist work on EDs (see Eckermann, 2009; Malson, 2009). In particular, it stressed how such work has situated anorexia and bulimia as discursive constructions - 'illnesses' and categories that 
are actively constituted by medical discourse. It then set out some of the main themes emerging from this research, especially with respect to why females may constitute the majority of ED sufferers. This covered such themes as 'The deeper meanings of the female body and its shape'; 'How women/girls are given messages about "appetite” in society'; 'How EDs might be used to negotiate the changing nature of womens' roles'; as well as the ways in which EDs have been linked to 'More acute forms of female oppression/ exploitation' (such as sexual or domestic abuse).

At the start of the interview, I provided a brief description of the study, and chose to disclose my own history of anorexia from which I recovered in 2009-10 (after 20 years). I explained how my engagement with the feminist literature since this time had prompted me to think about its role within treatment contexts, but I did not elaborate further in this regard and thus share my investment in the feminist critique of the biomedical model (and how it had enabled me to reassess what my ED 'was'). I was concerned that such information would make it difficult for participants to respond openly to the feminist ideas in a context already structured by different dimensions of power (Maynard and Purvis, 1994; Tang, 2002). Indeed, there is a considerable body of literature on the methodological and political complexities of women interviewing women (see Tang, 2002; Broom et al, 2009), and the debates raised by this research are clearly relevant here. Firstly, my decision to self-disclose was in part prompted by the long-running debate about the interviewer-interviewee relationship and the power dynamics in which the interview takes place. In critiquing a positivist epistemology, feminist qualitative research has invested in models which seeks to reduce the gap between 'expert' and participant (Oakley, 1981; Piran, 2010; Tracy, 2013), and has thus often included discussions of personal experience and reflexivity (Tang, 2002; Broom et al, 2009). But the possibility of a 'non-hierarchical' (Oakley, 1981) relationship (as 
based on gender congruence and perceptions of shared experience) has long been subject to critique, and feminist work has explored the range of social attributes - such as class, race, age and educational capital - that can shape the balance of power within the interview encounter (Tang, 2002; Broom et al, 2009). Indeed, as Sarah Tracy summarises, feminists believe that 'the researcher has a moral responsibility to be aware of their own power, the potential for its abuse, and issues of reciprocity [original emphasis]' (2013, p.56).

In this regard, self-disclosure becomes an ethical issue within the interview context, both in terms of establishing a potential rapport with participants, and with respect to thinking through the implications of personal admissions (Broom et al, 2009, p.53). For example, in willingly also submitting to the 'risks' of self-exposure, my self-disclosure was offered as a form of reciprocity (see Ribbens, 1989, p. 594), whilst it was also prompted by what felt congruent and authentic to me as a researcher: presenting as if I had no personal experience of an ED would have felt deeply uncomfortable and unethical. I also emphasised my readiness to answer any questions about my own experience of anorexia, an offer which several of the participants took up. In this respect, the disclosure of my history was repeatedly received in positive terms - whether this was expressed in the interview itself or via subsequent email contact - and over half of the participants claimed that they had rarely spoken about their experiences with another ED sufferer before. As such, whilst it would be problematic to characterise the interviews as simply 'friendly free-flowing discussion[s]' (Tracy, 2013, p. 56), they emerged, at times, as focused conversations (Spitzack, 1988, p.55) in which we shared treatment experiences, painful recollections and narratives of recovery.

At the same time, I also acknowledge that perceptions of gender congruence can be both productive and limiting (Broom et al, 2009, p. 53). Participants may have felt more obliged to 
offer favourable readings of the feminist material precisely because of gender congruence particularly given the nature of my personal disclosure (and thus experience of long-term suffering from anorexia). Furthermore, all interviews are shaped by norms of social desirability which may include - but are not limited to - the social conventions of politeness, particularly in encounters with unknown people (Williams and Heikes, 1993; Hewitt, 2007). Moreover, given that 'gender permeates all aspects of social life and the qualitative interview involves processes of performance and impression management' (Broom et al, 2009, p. 52), it has been argued that social desirability expression is itself gendered (Williams and Heikes, 1993), with social norms of femininity placing a particular premium on identities that are socially pleasing (Bartky, 1988). Indeed, it is precisely such social norms that the feminist approaches to EDs seek to elucidate, so it is thus important to acknowledge that there is no space 'outside' of such discourses in which this research and analysis can take place.

The provision of the feminist summary sheet is also clearly relevant when thinking about the power dynamics of the interview encounters, and the situated nature of the data they produced. My decision to use this sheet was explicitly shaped by a frustration with existing feminist qualitative work on EDs. Such research has often listened carefully, sensitively and reflexively to the voices of those diagnosed with anorexia (McSween, 1995; Malson, 1998; Saukko, 2008), and to a lesser extent bulimia (Burns, 2004). But it has not offered participants a space from which respond to the feminist conceptions of their problem. Rather, such voices are used by the researcher to interpret the role played by gender, usually after the interaction has taken place. In contrast, I wanted to explicitly involve the participants in conversations about the feminist research, and its various implications for their selfconceptions - an aim that can be situated in relation to wider work which has offered girls/ women the opportunity to respond to feminist conceptions of their identities (from beauty practices to veiling) (Baumgardner and Richards, 2010, Zine, 2006). The summary sheet was 
central to this intervention, but I also acknowledge here that it offered an imperfect solution to an epistemological and methodological problematic, in so far as I wanted to be able to ask participants about their views on perspectives that were possibly not central to their treatment experiences. This clearly meant that participants had to rely upon my summary of a field which was inevitably partial and subjective, whilst they were also being asked to give me their views on the document in my presence. Furthermore, although this did not constitute the first encounter with the feminist ideas for all of the participants, the very act of writing the sheet positioned me as the 'expert' in the encounter, both in terms of the academic ideas, and with regard to a complex and developed understanding of what 'we' had experienced. Although it is important to emphasise that some of the participants related gendered explanations of their problems which they had clearly held for some time, it is also possible that participants may have offered different understandings of their EDs if I had asked them to share their stories prior to the provision of the summary.

\section{Data analysis}

The interviews were recorded on a digital voice recorder and transcribed, and the participant data was anonymised at the point of writing. ${ }^{3}$ Informed by wider feminist qualitative research on EDs (e.g Burns, 2004; Boughtwood and Halse, 2010), I then conducted a thematic discourse analysis of the transcripts, which involved three stages. Upon a first read through the transcripts I deduced that the responses clustered around three broad themes (as extrapolated from the interview questions) which were 1) the womens' understanding of why they had developed an ED; 2) their evaluation of their treatment; and 3) their responses to the feminist perspectives. I then conducted an in-depth qualitative analysis of these three themes (Smith et al, 1995), looking for thematic patterns and commonalities. Lastly, I critically reflected on how the women positioned themselves in relation to the dominant discourses of 
medical treatment for EDs, and the feminist approaches which actively critique these discourses.

As Paula Saukko (2008) describes, discourse analysis does not unproblematically give participants 'voice', and in the context of ED research, she points to a potential tension between the desire to 'listen carefully and faithfully' to the women's experiences of eating and body distress, and the impetus to critically assess the discourses ... from which their voices are made...' (2008, p. 77). My article aims to raise questions about how this process has been pursued in feminist work, in so far as the participants are not given a chance to evaluate the critical approaches through which their identities are understood. Yet I am fully aware that I am still subjecting the womens' responses to critical and 'expert' analysis here, and that this research cannot exist outside of such a hierarchical framework. Nevertheless, I sought to create a space in which dialogue about the feminist approaches was enabled, albeit it in particular, situated ways.

\section{Results and Discussion}

\section{Approaching 'womanhood': Stories of the self}

Although to differing degrees, all of the participants in the study felt that the aetiology and significance of their ED related to their experience of gender and/or sexuality as women. Some of the respondents claimed to hold this view quite firmly prior to contact with my research project, whilst others felt that the summary sheet had 'resonated' with them and prompted them to reassess understandings of their eating/body distress in particular ways. Further, in ways similar to other qualitative studies (MacSween, 1995; Malson, 1998; Saukko, 2008), the stories articulated by the respondents were often presented in terms of a negotiation with - rather than a simple conformity to - what was perceived to represent 
dominant femininity: an identity that was white, slim, 'feminine' and heterosexual. Most of the participants in the study developed an ED during puberty. In reflecting back on this time, several spoke of somehow 'not fitting' with the perception of a dominant female norm, whether this was explained in terms of ethnic difference, a more fluid experience of sexuality, or perceivably 'masculine' traits - such as high intelligence or a 'tom-boy' identity. In this regard, it is notable that the heterosexual participants and the bisexual bi/pansexual participants spoke of the alienating nature of dominant norms of femininity in similar ways, even though the latter were effectively negotiating the constraints and pressures of heteronormativity, rather than exclusively heterosexual femininity. So in speaking about her sexual desire for girls as well as boys, a 19 year old with experience of anorexia and BED and who identified as bi-pansexual - explained how her apparent 'deviation' from heterosexual norms made her 'feel unworthy, different, just not fitting and not [measuring] up to the kind of girl or woman that I should have been' (P1). Similarly, a heterosexual participant with experience of anorexia explained:

I was not the social ideal and I knew it ... I wasn't a fat child but I was roly-poly and I had short hair... and that was snowballed by the fact that I [was]... highly intelligent. I would never compromise the intelligence so I ended up crippling myself in terms of my body because I thought well maybe if I just look like [the other girls] - if I look like gorgeous and thin - then maybe people will stop caring that I'm also really smart (P2).

An ED is presented here as a negotiation with dominant social norms of femininity, functioning as a strategy through which to retain or hide what both participants presented as 'less traditionally feminine' (P2) attributes of their identity. 
Others spoke more specifically of their ED as an attempt to evade what were seen as the expectations and experiences associated with adult femininity, a perception that has been explored in both medical and feminist literature on EDs (see Saukko, 2008). This evasion or refusal of adult heterosexual femininity was often discussed openly by the participants and understood as such. As one participant who suffered from anorexia for 25 years asserted: 'I didn't want to be a woman. I didn't want to be female. It's not that I wanted to be a boy. I just didn't want to be anything to do with being a woman ... breasts, babies, marriage, sex, men' (P8). Some participants vividly recollected experiences of bodily objectification in their teenage years, and presented the ED as a means to evade such visibility (see Malson, 2009), and to thus retain control of their bodily form. Others related their EDs to what they saw as the specific expectations and constructs surrounding contemporary femininity. One participant for example spoke of her 'use' of anorexia as a form of 'endurance training' - that not eating whilst doing her A-level exams would be like undertaking them with 'a ruck-sack on $[$ her] ... back. In response to my question, 'but what were you training for?' she explained:

Um, ... being a woman? The fact that so many opportunities are being opened up to women is great... but there are lots of ... conflicting demands and I think I felt a lot of pressure with those opportunities. ... I also had the sense that those things wouldn't be that easy to access and I'd still have to fight for them... I was trying to fight for some kind of independence but still trying to achieve (P12).

The participant seems to nod to a pressure to adhere to a postfeminist life script in which the attainment of such 'opportunities' are seen as compulsory and 'natural', whilst she also points to the potential barriers that might make access to this identity both difficult and unequal. 
As discussed earlier in the article, there are a number of qualitative studies which have used feminist approaches to understand how girls/ women articulate their experience of having an $\mathrm{ED}$, and the discursive complexities these articulations involve (McSween, 1995; Malson, 1998, 2009; Burns, 2004; Saukko, 2008). I thus do not develop the discussion of the women's stories further here, in part due to issues of space, but also because the article is interested in how such understandings can be situated in relation to treatment experiences, as well as the womens' evaluations of the feminist approaches themselves.

\section{'She would just look at me like I was barmy': feminist discourses in treatment}

The participants had collectively encountered a wide range of treatments in the UK. This included various forms of therapy (which ranged across CBT, psychodynamic therapy and what was often simply described as 'talking therapy'), as well as hospital admissions and different forms of ED treatments within out-patient and in-patient contexts. With regard to anorexia at least, the current NICE guidelines advocate the inclusion of treatment which considers ‘wider psychosocial issues [related to]... the expectation of weight gain' (2004). But it was clear that none of the participants felt that such issues, which must surely include gender, formed a significant or continuous part of their treatment experiences.

One respondent with experience of long-term bulimia who had engaged with a community ED service and two years of 'talking therapy' explained:

In my treatment it was never really raised about gender. I think there was some talk about media images but even this wasn't [related to] gender specifically.... I was just told, um, to try and not notice images of bodies... It's a bit ridiculous really (P9). 
Relating back to criticisms of body image work and the ways in which this may abstract media images from the wider contexts of gender inequity (Piran, 2010), the participant recounted what she received as somewhat preposterous advice. In other participant accounts, any emphasis on 'culture' or 'gender' was just recalled as absent. The idea of treatment inadequately recognising what was felt to be the significance of femininity /sexuality was encapsulated by one participant who had experience of both anorexia and bulimia and who felt that 'gender was a huge part of [her]... experience of having an ED' (P3). The participant primarily held a positive view of the female clinical psychologist she had worked with, but also explained how it was only after a near successful suicide attempt - prompted by the recommencement of her periods - that the psychologist said 'Right we need to talk about this, and [she] really pushed me.... It ... was then that somebody actually clicked that there was more going on than people had picked up on' (P.3).

Others felt that gender was focused on to a very limited degree and explained how it was entirely up to them to introduce the topic as significant. Three of the respondents had come across aspects of the feminist literature outside of their immediate treatment contexts and asked if they could incorporate it into their therapy. As one respondent who had been in a specialist in-patient unit for five months explained:

When I was reading [Orbach's Hunger Strike]... I tried bringing it up with the psychiatrist ... She would just look at me like I was barmy - like that was more madness coming from me...I was quite confused about it but it was like 'OK let's put [the book]... away again: that's just [name removed] being crazy again...' (P5).

The participant explained how she indeed stopped reading the book that she had initially judged as so resonant. This was partly because it destabilised the gender norms that she had 
'been brought up to believe and that society was telling me to believe,' but also because she could not reconcile the approach of the book with her wider treatment regime. As she explained: 'It didn't tie up with what else was being said to me [about anorexia]'.

Almost half of the participants in the sample had been admitted for in-patient treatment, whether this was to hospitals or specialist centres. But consolidating wider feminist critiques of such interventions, (Sesan, 1994; Gremillion, 2003), it was these contexts that were positioned as the most antithetical to the exploration of feminist ideas. Talking specifically of an adolescent in-patient unit which withheld one-to-one therapy until a baseline weight was reached, one participant with experience of anorexia described how:

It is only once you learnt that kind of submissive femininity where you don't protest and you don't seek 'excessive' independence or your own views that you'd be kind of granted the right to speak again, although on, like, really limited terms (P12).

Although the idea of the tiny, fragile and starved body might be read as a form of submissive femininity in itself (adhering to traditional cultural scripts of gender in which women should take up less 'masculine’ space (Orbach 1986; Wolf 1991)), critical feminist work has also interpreted it as a form of corporeal resistance, or a rejection of traditional heterosexual femininity/ feminine subjectivity (Bordo 1993; Malson 2009; Saukko 2008). The suggestion of the anorexic body (and voice) as resistant and thus in need of 'discipline' is implicit in the participant's response above, and the descriptions of in-patient treatment which emerged in the study often described environments that were oppressive and punitive. Such recollections run entirely counter to Robin Sesan's suggestion that, because inpatient treatment settings often provide a 'predominantly female community for patients', a 'feminist component is 
potentially built in...' (1994, p.261). Whilst such settings were described as predominantly female by the participants - and sometimes warmly so - such political possibilities were very far from realised.

\section{'[I]t seems so bloomin'obvious to me': evaluating feminist approaches to EDs}

I fully recognise that there were potential power dynamics within the interview context which may have shaped the ways in which the feminist material was evaluated. But the feminist ideas were nevertheless discussed in highly positive ways, with the responses moving from what I saw as very high levels of affirmation (11) to partial endorsements, which contained questions and reservations (4). Furthermore, it is crucial to recognise the ways in which the responses to these approaches were inevitably bound up with the participants' views on 'feminism' itself, as articulated in an era in which many young women are perceived to be disaffected from feminist politics, as shaped by discourses of generationalism, post-feminism and neoliberal ideologies of individualism (see Scharff, 2012). Although I did not ask the participants to discuss their relationships with feminism per se, some of them declared themselves to be proudly 'feminist', whilst others occupied more ambivalent positons in which feminism was both claimed and distanced.

Firstly, just as the medical approaches have presented themselves as 'common sense', one of the most recurrent responses to the feminist approaches was that they too seemed to have an 'obvious' explanatory power. As one respondent suggested: 'I ... feel exasperation really as it seems so bloomin' obvious to me ... I can't understand ... why [gender was] ... not explored more explicitly in my treatment' (P8). Another participant, who came across the feminist literature in her first year at university, expressed rather more than exasperation when she stated: 
Like why hadn't I come across this sooner? I felt like it had been deliberately withheld from me... I think there is an element of truth in that. I think [the feminist work]... is such a threat to the medical establishment because it threatens their power over you (P12).

The use of the term 'deliberately withheld' suggests a feeling in which the feminist literature was not simply omitted, but effectively censored.

Secondly, the feminist approaches were frequently described as a welcome challenge to the medical emphasis on EDs as a form of individual pathology. As one respondent with experience of anorexia explained:

It would have been a whole lot easier if these [feminist] perspectives were used in my treatment... It always felt like me against the system which is really isolating....I would have seen that it wasn't just me being crazy...The idea of eating disorders being a problem of society I actually found really reassuring ... rather than just you've gotta sort yourself out and fit back into the society structure that we've got (P5).

The shift away from the ('crazy') individual to seeing one's subjectivity as shaped by wider social structures is framed as empowering here, whilst it is also positioned as potentially facilitating a distance from the ED itself.

But in other ways, the perceived collectivism of feminism emerged as a tension within the responses. In speaking to a long-running debate about the extent to which feminism might be seen as exclusionary (not speaking for ' $m e$ ') as well as homogenising (making assumptions of my 'behalf'), a minority of the participants questioned what they saw as the implicit power 
dynamics at work in the approaches and the ways in which they positioned them as gendered subjects. In this regard, it is important to emphasise that I did not simply ask the women what they thought of the ideas on the summary sheet: I also asked them how they felt when reading them, in the hope that this might make space for affective responses - such as anger, frustration, shock or recognition - rather than simply cognitive reactions.

One of the younger participants in the study (19) suggested that the sheet 'set up a number of points that like did really resonate with me', but that the 'personal part of me ... was also quite angry ... defensive. Like it made me feel a bit oppressed and diminutive' (P1). Another recalled reading the summary sheet in the presence of her flatmate and described how she said out loud: 'I'm reading this and I'm not sure I agree with it. It is saying ... that developing an eating disorder is a symptom of ... a patriarchal society! [laughs]' (P4). As discussed earlier in the article, although participant research has been central to feminist scholarship on EDs, those with experience of an ED are not conventionally asked to respond to the feminist critical frameworks through which their voices are understood. In this regard, such participant critiques are important, raising long-standing questions about the epistemological project of feminism, and its relationship to the subjectivities of the women its researches (Maynard and Purvis, 1994; Skeggs, 2003). Although the feminist work does not simply position women who develop EDs as 'victims' of gender inequality (or patriarchal 'false consciousness'), it is important that the feminist discourses were partly experienced as such by some of the participants. It was not that gender was seen as irrelevant to their relationship with an ED - far from it - but that such participants wanted to enter into a dialogue about these political possibilities, and have a voice in their potential exploration. 
A further site of tension emerged in relation to the feminist suggestion that 'eating disorders and disordered eating form a continuum among women' (Liimakka, 2013, p. 22), and that EDs should be positioned within the context of normative femininities. The claim that EDs are inextricably linked to the everyday construction of femininity has also been the aspect of the feminist scholarship most attacked by the biomedical model: it is seen to deny the severity of anorexia and bulimia (which are not diets gone 'wrong'), whilst failing to explain why not all women develop such problems acutely (see Bordo, 1993, p. 52). Responses to these criticisms have been addressed eloquently elsewhere (Ibid, pp.60-1), but for a minority in the sample (4), this idea of a continuum was not seen as helpful nor enabling. As one respondent with experience of anorexia explained:

I don't want eating disorders ... to be on a spectrum with basically what it is to be feminine. ... I guess I am quibbling with the feminist theorists on that... but that almost makes me feel more stigmatised as someone who suffered from it, like why wasn't I on the 'normal' part of the spectrum? (P2).

Critical feminist work has fought against what it sees as the stigmatising consequences of EDs being labelled as mental illnesses. But the respondent above regards being on a 'normative' scale of body/eating distress to be pathologising in itself, both for women in general and with regard to her own experience of anorexia. A number of the participants were in fact critical of the positioning of EDs as mental illnesses, suggesting that, despite apparent changes in public perceptions of mental health problems, the 'stigma is still massive' (P1). Yet there was still a sense in which some preferred the labelling of EDs as mental health/ psychiatric disorders because they facilitated a discourse through which their problems could be taken seriously. The responses which partially supported this view (4 out of 15) should be seen as representing the felt reactions of these participants. At the same time, such 
perceptions cannot be separated from the hegemony of psychiatric and medical discourses in the treatment of EDs more widely, and the ways in which medicine - as opposed to 'culture' connotes legitimacy, expertise and power (Foucault, 1973). This is particularly so given that - in both popular media and medical discourse - the role of culture in the production of EDs is often reduced to the media, diet and fashion industries (Malson, 2009: 135). Such a construction not only locates EDs within discourses of narcissism and vanity, but it also situates sufferers as 'vulnerable' media consumers (designating a subject position that is not attractive to occupy).

Finally, one quarter of the women in the sample were concerned about the extent to which male EDs could be explained via, or benefit from, the feminist models. As one participant cautioned: 'I think the only thing that worries me about this is the boys, the men, like where would they fit?' (P5). Such a response may speak to the ways in which too stark an emphasis on inequality - and the idea of not being on an 'equal' footing with men - occupies a precarious role within contemporary young feminism. Yet given the rising number of boys/men being diagnosed with EDs, as well as the extent to which such questions have been asked of the feminist scholarship more widely (Maine and Bunnell, 2008), these are also reasonable and pragmatic concerns. As a response to this perceived omission, I exchanged views with the participants about how an emphasis on cultural identity, including questions of embodied masculinity and sexuality, might also applied to male treatment, a debate which is already in evidence in research into male EDs (Strother et al, 2014). At the same time, the suggestion in such work that a cultural approach would need to be differentiated primarily because 'treatment paradigms have been geared toward females' (Ibid: 436), massively overestimates the existence of such gendered 'tailoring' in the first place.

\section{Imagining feminist approaches in treatment}


After discussions of how participants evaluated the feminist approaches, the interview explored whether/ how respondents thought they should be implemented. Although the relationship between the biomedical and feminist perspectives has been characterised as a 'paradigm clash' (Levine and Smolak, 2014), most of the participants did not see them as entirely antithetical. As one explained: 'I don't see the conflict about bringing gender into the treatment ... I feel like we are just adding an important layer to how we ... can explain our problems' (P2). Opinions on whether the feminist discourses might work most productively in a group or singular context were divided, and were clearly shaped by respondents' prior experiences of such settings in treatment. Some felt that the feminist approaches would only work in a one-on-one therapeutic context - if the therapist were appropriately trained within this sphere - especially given that it might involve sensitive discussions of sexuality, sexual experiences and parental role models. This reflects back on the history of individual feminist therapists working with ED sufferers (Orbach, 1979, 1986, Lawrence, 1984), which is the primary way in which the relationship between EDs and feminist approaches have been discussed. Others, however, felt that a collective dialogue was central for the exploration of the ideologies that the feminist approaches were seeking to deconstruct, and we discussed the possibilities of treatment groups that would bring feminist perspectives to bear on issues of gendered bodily norms, the construction of female 'appetite' in society, and the ways in which EDs might be situated on a continuum with normative femininities. Foregrounding the importance of collective dialogue, one participant explained how:

I think it would work better in a group because then you would have multiple experiences of being feminine or being a woman on display, so that we can identify the core generalisations where we are kind of going for some supposed norm or ideal... (P2). 
In explicitly framing the idea as a means through individual women can come together to recognise and realise aspects of collective struggle, this response has much in common with the discursive construction of consciousness-raising groups (as linked in particular to second wave feminism). As discussed earlier in the article, there are some isolated examples of such groups flourishing within particular treatment contexts, but they are not routinely part of mainstream treatment.

Other disagreements unfolded across what stage of the treatment process feminist interventions might be most usefully realised. This article has already discussed how, in the context of anorexia, withholding thinking and talking treatments until a base level BMI is reached can be seen as both punitive and silencing from a feminist point of view. But some participants did feel that the feminist approaches might be put to best use during recovery:

I think for me it is more about the recovery... more about the time after the initial crisis... And the problem that I had is that I didn't really feel like [treatment] ... equipped me to live in the world as a recovered person sufficiently to stop me relapsing ... When my eating became normalised and I experienced having a physical presence in the world ... my understanding of myself in that world was not robust enough... [original emphasis] (P11).

This respondent was diagnosed with anorexia at age 11, so developing a 'womanly' body was only confronted during recovery. In describing how she suddenly felt that she had a 'physical' or corporeal presence in the world, the participant was referring to the experience of objectification, or what she described as feeling 'visibly and publicly sexual'. Concurring with research which suggests that a feminist identity may play some role in countering feelings of both objectification and body dissatisfaction (Murnen and Smolak, 2009; Rubin et 
al, 2004), she went on to suggest that embracing feminism at university (with a group of likeminded friends) enabled her to 'understand' the feelings and provided an 'outlet' for her anger.

Finally, given that this participant encountered both feminism and the feminist work on EDs at university, this response foregrounds issues of access and inclusion. As acknowledged in the outline of the sample, whilst the participants came from a range of socio-economic backgrounds, all had been to university, and thus arguably possessed particular levels of educational capital. The extent to which factors such as class and education might regulate feminism's perceived accessibility has been widely debated (Skeggs, 2003). This was also an area of concern for some of the participants who were in fact self-reflexive about the ways in which their educational and cultural experiences had enabled them to encounter aspects of the feminist debates, or firmly relate them to their self-understandings. As one commented: 'People who wouldn't have feminist friends who were sending them those things or have access to that might not encounter [these debates]... on their own' (P7). Another expressed how:

It does make me feel quite angry that it's not more widely discussed... It seems like it's a bit hidden really... It's like you have to be educated, something that you have to ... seek ... out, or come across at university (P9).

Both responses express concern over the accessibility of the feminist literature. Indeed, the fact that some of this work is intellectually dense and complex cannot be ignored. But in imagining feminist treatment scenarios in practice, none of the participants endorsed the idea of didactic 'sessions' in which they were handed a 'pamphlet saying "did you know you are subjugated by a male gaze?"' (P11). Rather, we spoke about how ideas distilled from the feminist theories might function within clinical settings. It is also important to recognise here 
that the comments made by P7 and P9 above focus less on the idea of feminism as an exclusionary movement - associated only with educated and privileged women - than they also draw attention to how such approaches have been effectively confined to educational establishments, in part dictating who has access to their possibilities.

\section{Discussion and conclusion}

In 1992 Marta Robertson observed how the biomedical model 'crushes impulses to search out different ways of understanding' EDs (1992, p. xi). Although critical feminist work on EDs has grown exponentially since this time, developing a sophisticated and convincing language through which to talk back to these prevailing discourses, Robertson's statement may remain entirely apposite for those classed as 'patients'. Whilst the women in this study articulated a range of responses to the feminist approaches - some of which flag up tensions or problems which a practical application of such perspectives could seek to address - all expressed the sense that they would likely have been helpful in treatment, and many fervently so. Based on the responses here, it is clear that any focus on gender in treatment was either peripheral or invalidated, and certainly had to come from the women themselves. From a feminist perspective this is both worrying and frustrating: with regard to what other 'illnesses' do we expect sufferers to set an appropriate agenda for their own treatment?

Although there has historically been something of a split between research and practice in the treatment of EDs, the divide between critical feminist perspectives and treatment approaches should be seen as political. As Bordo summarises, the 'medical model has a deep professional, economic, and philosophical stake in preserving the integrity of what it has demarcated as its domain, and the result has been a frequent blindness to the obvious' (1993, 
p. 53). But what has arguably become lost or muted within the field of feminist work is what Sesan (1994) forcefully articulated in 1994 as a 'call to action!' to intervene in treatment practices. The feminist work on EDs never set out to be predominantly analytic in nature and - according to this study - nor should it be.

I also fully recognise the ways in which this research was prompted by the intersection between $m y$ identities as a former 'anorexic', feminist and academic, and the consequences of this for the production and analysis of the data. All qualitative data needs to be conceived as situated and shaped by the interpersonal dynamics of interview encounters (Broom et al, 2009 , p. 52), and I am happy to acknowledge my personal and political investment in this study here. I feel that a focus on questions of gender and sexuality would have been beneficial as part of my treatment for anorexia, and that my reasons for developing a longterm ED were profoundly imbricated within the normative structures of femininity. The feminist literature also makes a difference to me now, in terms of how I negotiate my relationship with female embodiment, every single day. But it is also important not to qualify the impact of my own subjectivity here to a point in which the participant responses cease to matter. The women in the study collectively spoke of a mismatch between their understanding of their EDs and their treatment, and such disparities experiences should not be dismissed or ignored. Furthermore, although the demography of the sample tends toward the socially normative - predominantly white, educated and heterosexual - it is also the imagined bodies, minds and identities of this group upon which much of the biomedical model has been 'built' (especially with regard to construction of anorexia).

Debates about treatment efficacy rarely paint an optimistic picture, whilst the numbers of people diagnosed with anorexia or bulimia continue to rise (Malson et al, 2004). Within this context, it seems important to (re)start a conversation about the role of gender identity in the 
treatment of anorexia and bulimia, and it was often simply a 'conversation' that the women in this study wanted to have. Anorexia and bulimia are often the source of unimaginable pain, loss, suffering and distress. Is a conversation really too much to ask?

\section{Acknowledgements}

With sincere thanks to Beat for assisting with the recruitment process, to the participants who shared their stories and opinions with me, and to Sanna Inthorn for helpful comments on the article.

\footnotetext{
${ }^{1}$ In this regard, the feminist suggestion that EDs are a manifestation of the pressures and contradictions associated with normative femininities takes in the range of discursive contexts in which gender is produced, including the family, the media, the education system, consumerism and so on.
}

${ }^{2}$ Feminist-focused programmes in other national contexts have also been discussed, such as the example of EDEN in New Zealand (see Burns et al, 2009).

${ }^{3}$ I have replaced names with a participant number (i.e. P1) which reflects the order in which the interviews were transcribed.

${ }^{4}$ Such responses could be productively considered in relation to research on the cultural relationships between lesbianism and EDs. In their empirical study, Jones and Malson (2013) explore how EDs (and anorexia in particular) can indeed offer an attempt to eradicate lesbian subjectivity and desire, whilst presenting a 'way of "looking straight" by taking to 'extremes' the thin, heterosexual, female ideal (Jones and Malson, 2013: 68). 


\section{References}

Bartky, S. L. (1988). Foucault, Femininity, and the Modernization of Patriarchal Power. In I.

Diamond I and L. Quinby (Eds), Feminism and Foucault: Reflections on Resistance (pp.61-

86). Boston: Northeasten University Press.

Baumgardner, J and Richards, A. (2010). Manifesta: Young Women, Feminism and the

Future, $10^{\text {th }}$ anniversary ed. New York: Farrar Straus Giroux.

Bordo, S. (1993). Unbearable Weight: Feminism, Western Culture and the Body. London and California: University of California Press.

Boskind-White, M. (1979). Cinderella's Stepsisters: A feminist perspective on anorexia nervosa and bulimia. In J.H Williams (Ed), Psychology of Women (pp12-25), New York: W.W Norton.

Boughtwood, D and Halse, C. (2010). Other than Obedient: Girls' Constructions of Doctors and Treatment Regimes for Anorexia Nervosa, Journal of Community and Applied Psychology, 20 (2): 83-94.

Broom, A, Hand, K and Tovey, P. (2009). The role of gender, environment and individual biography in shaping qualitative interview data, International Journal of Social Research Methodology, 12 (1): 51-65.

Burkitt, J. S. I (2001) Embodiment, Lived experience and anorexia: the contribution of phenomenology to a critical therapeutic approach, Athena Digital, 0 April: 38-52.

Burns, M. (2004). Eating like an Ox: Femininity and Dualistic Constructions of Bulimia and Anorexia, Feminism and Psychology, 14 (2): 269-295. 
Burns, M. (2009). Bodies as (im)material? Bulimia and body image discourse. In H. Malson and M. Burns (Eds), Critical Feminist Approaches to eating dis/orders (pp124-134), Sussex: Routledge.

Burns, M, Tryer, J and The Eating Difficulties Education Network (EDEN). (2009).

Feminisms in Practice: Challenges and opportunities for an eating issues community agency.

In H. Malson and M. Burns (Eds), Critical Feminist Approaches to eating dis/orders, (pp221231), Sussex: Routledge.

Chernin, K. (1985). The Hungry Self: Women, Eating and Identity. New York: Harper Collins.

Diagnostic and Statistical Manual of Mental Disorders V5. 2013.

Foucault, M. 1973. The Birth of the Clinic: An Archaeology of Medical Perception, New York: Pantheon Books.

Gremillion, H. (2003). Feeding Anorexia: Gender and Power at a Treatment Center, Durham and London: Duke UP.

Hewitt, J. (2007). Ethical Components of Researcher-Researched Relationships in Qualitative Interviewing, Qualitative Health Research, 17 (8): 1149-1159

Holstrom, A. (2004). The Effects of the Media on Body Image: A Meta-Analysis. Journal of Broadcasting and Electronic Media, 48 (2): 196-217.

Houston-Grey, S. (2011). 'A Perfect Loathing: The Feminist Expulsion of the Eating Disorder', .KB Journal, 7 (2) http://kbjournal.org/grey. Lawrence, M. (1984). The Anorexic Experience. London: The Women's Press. 
Jones, R; Malson, H (2013) A critical exploration of lesbian perspectives on eating disorders. Psychology and Sexuality 4 (1): 62-74.

Lee, S. (2004). Engaging Culture: An Overdue Task for Eating Disorders Research, Culture, Medicine and Psychiatry, 28 (4): 617-621.

Levendusky, P.G and Dooley, C.P. (1985). An Inpatient Model for the Treatment of Anorexia Nervosa, Social Science and Medicine, 44 (4): 479-89.

Levine, M and Smolak, L. 2014. Paradigm clash in the field of eating disorders: a critical examination of the biopsychiatric from a sociocultural perspective, Advances in Eating Disorders: Theory, Research, Practice, 2 (2): 158-170.

Levine, M and Maine, M. (2010). Are media an important medium for clinicians?: mass media, eating disorders and the Bolder model of treatment, prevention and advocacy. In $\mathrm{M}$. Maine, Beth H. McGilley, and D.W Bunnell (Eds), Treatment of eating disorders: Bridging the Research Practice Gap (pp.53-67), Academic press, Burlington MA.

Liimakka, S. (2013). Re-embodied: Young Women, the Body Quest and Agency in the Culture of Appearances, Academic Dissertation, found at https://helda.helsinki.fi/bitstream/handle/10138/38047/reembodi.pdf?sequence=1 Maine, M and Bunnell, D. (2008). How do the Principles of the feminist, relational model apply to treatment of men with eating disorders and related issues?, Eating Disorders: The Journal of Treatment and Prevention 16 (2): 187-192.

McSween, M. (1995). Anorexic Bodies: A Feminist and Sociological Perspective on Anorexia Nervosa. London: Routledge. 
Malson, H. (1998). The Thin Woman: Feminism, post-structuralism and the social psychology of anorexia nervosa. London: Routledge.

Malson, H. (2009). Appearing to disappear: Postmodern Femininities and self-starved subjectivities. In H. Malson, and M. Burns (Eds), Critical Feminist Approaches to eating dis/orders, (pp.135-145), Sussex: Routledge.

Malson, H, Finn, D.M, Treasure, J, Clarke, S, Anderson, G. (2004). Constructing 'The Eating Disordered Patient': A Discourse Analysis of Accounts of Treatment Experiences, Journal of Community and Applied Psychology, 14 (6): 471-489.

Malson H, Burns, M (2009) Re-theorising the slash of dis-order: An introduction to critical feminist approaches to eating dis/orders. In H. Malson and M. Burns, M (Eds), Critical Feminist Approaches to eating dis/orders, (pp.1-6), Sussex: Routledge.

Malson, H and Ryan, V. (2008). Tracing a Matrix of Gender: An Analysis of the Feminine in Hospital-Based Treatment for Eating Disorders, Feminism and Psychology, 18 (1): 112-132.

Maynard. M and Purvis. J (Eds). (1994). Researching Women's Lives from a Feminist Perspective, London: Taylor and Francis.

Maynard, M. (1994). Methods, Practice and Epistemology: The Debate about Feminism and Research. In M. Maynard and J. Purvis (Eds), Researching Women's Lives from a Feminist Perspective, (pp.10-26), London: Taylor and Francis.

Murnen, S.K and Smolak, L. (2009). Are feminist women protected from body image problems? A meta-analytic review of relevant research, Sex Roles, 60 (4): 186-197. NHS Choices, Eating Disorders (2015) http://www.nhs.uk/conditions/eatingdisorders/pages/introduction.aspx. 
NICE. National Institute for Health and Care Excellence. (2004). Eating disorders: Core interventions in the treatment and management of anorexia nervosa, bulimia nervosa and related eating disorders, https://www.nice.org.uk/guidance/cg9

Oakley, A. (1981). Interviewing Women: A Contradiction in Terms. In H. Roberts (Ed.), Doing Feminist Research, (pp.31-61). London: Routledge and Kegan Paul.

Orbach. S. (1978). Fat is a Feminist Issue, London: Arrow Books.

Orbach. (1986). Hunger Strike: the Anorectic's Struggle as a Metaphor for Our Age. London: Faber \& Faber.

Piran, N. (2010). A feminist perspective on risk factor research and on the prevention of eating disorders. Eating disorders: The Journal of Treatment and Prevention, 18 (3), 183 198.

Ribbens, J. (1989). Interviewing - An 'Unnatural' Situation?, Womens Studies International Forum, 12 (6): 579-92.

Robertson, M. (1992). Starving in the Silences: An Exploration of Anorexia Nervosa, Sydney: Allen and Unwin.

Roth, D. (1986). Treatment of the Hospitalized Eating Disordered Patient, Occupational Therapy in Mental Health, 6 (1): 67-87

Rubin, L.R, Nemeroff, C.J and Felipe Russo, N. (2004). Exploring Feminist Women's Body Consciousness, Psychology of Women, 28 (1): 27-37.

Sastre, A. (2014). Towards a radical body positive: Reading the online 'body positive movement', Feminist Media Studies, 14 (6): 929-943.

Saukko, P. (2008). The Anorexic Self: A personal and Political Analysis of a Diagnostic Discourse. Albany: State University of New York Press. 
Scharff, C. (2012). Repudiating Feminism: Young Women in a Neoliberal World, Farnham, Ashgate.

Sesan. R. (1994). Feminist Inpatient treatment for eating disorders: An oxymoron?. In P. Fallon, M.A Katzman, S.C Wooley (Eds), Feminist Perspectives on Eating Disorders (pp.251-72). New York and London: The Guildford Press.

Skeggs, B (1997) Formations of Class and Gender: Becoming Respectable. London: Sage.

Skoger, U.E and Magnusson, E. (2015). What makes feminist knowledge legitimate for therapists? A study of Swedish child psychotherapists. Feminism and Psychology, 25 (4): 489-505.

Smith, J.A, Harre, R and Van Langenhare, L. (1995). Rethinking Methods in Psychology, London: Sage.

Spitzack, C. (1988). Body Talk: The Politics of Weight Loss and Female Identity. In B. Bate and A. Taylor (Eds.), Women Communicating: Studies of Women's Talk (pp.51-74), Ablex Publishing: New Jersey.

Squire, S. (2003) Anorexia and Bulimia: Purity and Danger, Australian Feminist Studies, 18 (40): $17-26$.

Stice, E, Shaw, H, Becker, C Rohde, P. (2008) Dissonance-based Interventions for the Prevention of Eating Disorders: Using Persuasion Principles to Promote Health, Prev Sci, 9(2): 114-128.

Strother, E, Lemberg, R, Chariese Stanford'S and Turberville, D. (2014). Eating Disorders in Men: Underdiagnosed, Undertreated, and Misunderstood, Eating Disorders: The Journal of Treatment and Prevention, 20 (5): 346-355 
Tang, N. (2002). Interviewer and Interviewee Relationships Between Women, Sociology, 36 (3): 703-721.

Tracy, S.J. (2013). Qualitative Research Methods. Chichester: Wiley Blackwell.

Walsh, E and Malson, H. (2010). Discursive constructions of eating disorders: A story completion task, Feminism and Psychology, 20 (4): 529-537.

Warin. M (2010) Abject Relations: Everyday Worlds of Anorexia, New Brunswick: Rutgers.

Williams, C.L and Heikes, E.J. (1993). The importance of researcher gender in the in-depth interview: Evidence from two case studies of male nurses, Gender and Society 7 (2): 280-291

Wolf, N. (1991). The Beauty Myth: How Images of Beauty are Used Against Women, London: Vintage.

Zine, J. (2006). Unveiled Sentiments: Gendered Islamophobia and Experiences of Veiling among Muslim Girls in a Canadian Islamic School, Excellence and Equity in Education, 29 (3): 239-252. 